PROCEEDINGS OF THE

AMERICAN MATHEMATICAL SOCIETY

Volume 134, Number 12, December 2006, Pages 3703-3705

S 0002-9939(06)08417-6

Article electronically published on June 12, 2006

\title{
A SHORT PROOF OF A CONJECTURE ON THE CONNECTIVITY OF GRAPH COLORING COMPLEXES
}

\author{
ALEXANDER ENGSTRÖM \\ (Communicated by Paul Goerss)
}

\begin{abstract}
The Hom-complexes were introduced by Lovász to study topological obstructions to graph colorings. It was conjectured by Babson and Kozlov, and proved by Cukić and Kozlov, that $\operatorname{Hom}\left(G, K_{n}\right)$ is $(n-d-2)$-connected, where $d$ is the maximal degree of a vertex of $G$, and $n$ the number of colors. We give a short proof of the conjecture.
\end{abstract}

\section{INTRODUCTION}

It was conjectured by Babson and Kozlov [1, and proved by Čukić and Kozlov 4, that $\operatorname{Hom}\left(G, K_{n}\right)$ is $(n-d-2)$-connected, where $d$ is the maximal degree of a vertex of $G$, and $n$ the number of colors. We give a shorter proof of this, by generalizing the proof of that $\operatorname{Hom}\left(K_{m}, K_{n}\right)$ is $(n-m-1)$-connected in Babson and Kozlov [1].

For defintions and basic theorems on Hom-complexes used in this text, see the papers mentioned above, or the survey by Kozlov [6].

\section{An analogue of the Chromatic number}

An independent subset of vertices of a graph is a set, such that no vertices of it are adjacent. The minimal number of sets needed to partition the vertex set of a graph $G$ into independent sets is the chromatic number $\chi(G)$.

Definition 1.1. A covering $I_{1}, I_{2}, \ldots, I_{k}$ of $G$ is a sequence of independent subsets of $V(G)$ such that they partition $V(G)$, and $I_{i}$ is a maximal independent set in the induced subgraph of $G$ with vertex set $I_{i} \cup I_{i+1} \cup \ldots \cup I_{k}$, for all $i$, where $1 \leq i \leq k$.

A partition of $G$ into $\chi(G)$ independent sets can always be transformed to a covering by ordering the independent sets and if needed enlarging them. But a covering can use more than $\chi(G)$ sets. Define $\dot{\chi}(G)$ to be the maximal number of sets in a covering of $G$. Clearly, $\dot{\chi}(G) \geq \chi(G)$.

Lemma 1.2. If $d$ is the maximal degree of a vertex of $G$, then $\dot{\chi}(G) \leq d+1$.

Received by the editors May 31, 2005 and, in revised form, July 6, 2005.

2000 Mathematics Subject Classification. Primary 57M15, 05C15.

Key words and phrases. Graph homomorphisms, $k$-connectivity, Hom-complexes, graph coloring complexes.

This research was supported by ETH and Swiss National Science Foundation Grant PP002102738/1.

(C)2006 American Mathematical Society Reverts to public domain 28 years from publication 
Proof. Let $I_{1}, I_{2}, \ldots, I_{\dot{\chi}(G)}$ be a covering of $G$, and $v \in I_{\dot{\chi}(G)}$. For each $i$, where $1 \leq i<\dot{\chi}(G)$, there is a $w \in I_{i}$ adjacent to $v$, because otherwise $I_{i}$ would not be a maximal independent set. Hence the degree of $v$ is at least $\dot{\chi}(G)-1$. The degree of $v$ is at most $d$, thus $\dot{\chi}(G) \leq d+1$.

Lemma 1.3. If $H$ is an induced subgraph of $G$, then $\dot{\chi}(H) \leq \dot{\chi}(G)$.

Proof. It suffices to prove this when $H$ and $G$ only differ by a vertex $v$ of $G$. Let $I_{1}, I_{2}, \ldots, I_{\dot{\chi}(H)}$ be a covering of $H$. If $v$ is adjacent to a vertex in each of the sets $I_{i}$, then $\{v\}, I_{1}, I_{2}, \ldots, I_{\dot{\chi}(H)}$ is a covering of $G$ and $\dot{\chi}(H)+1 \leq \dot{\chi}(G)$. Otherwise, let $I_{j}$ be the first set in the covering such that $v$ is not adjacent to any vertex of $I_{j}$. Then $I_{1}, I_{2}, \ldots, I_{j} \cup\{v\}, \ldots, I_{\dot{\chi}(H)}$ is a covering of $G$, and $\dot{\chi}(H) \leq \dot{\chi}(G)$.

Lemma 1.4. If $I$ is a maximal independent set of $G$, then $\dot{\chi}(G)>\dot{\chi}(G \backslash I)$.

Proof. Let $I_{1}, I_{2}, \ldots, I_{\dot{\chi}(G \backslash I)}$ be a covering of $G \backslash I$. Then $I, I_{1}, I_{2}, \ldots, I_{\dot{\chi}(G \backslash I)}$ is a covering of $G$ with $1+\dot{\chi}(G \backslash I)$ sets.

\section{Higher Connectivity of $\operatorname{Hom}\left(G, K_{n}\right)$}

Lemma 2.1. If $I$ is an independent set of $G$, and $I^{\prime} \subset I$, then $\Delta=\{\eta \in$ $\left.\operatorname{Hom}\left(G, K_{n}\right) \mid n \in \eta(i) \Rightarrow i \in I\right\}$ collapses onto $\Delta^{\prime}=\left\{\eta \in \operatorname{Hom}\left(G \backslash\left(I \backslash I^{\prime}\right), K_{n}\right) \mid n \in\right.$ $\left.\eta(i) \Rightarrow i \in I^{\prime}\right\}$.

Proof. It suffices to prove this when $I \backslash I^{\prime}=\{v\}$. Let $\eta_{1}, \eta_{2}, \ldots, \eta_{k}$ be an ordering of $\{\eta \in \Delta \mid n \notin \eta(v)\}$ such that if $\eta(w) \supseteq \eta^{\prime}(w)$, for all $w \in V(G)$, then $\eta$ is not after $\eta^{\prime}$. Define $\eta_{i}^{*}$ as $\eta_{i}^{*}(w)=\eta_{i}(w)$ for $w \neq v$, and $\eta_{i}^{*}(v)=\eta_{i}(v) \cup\{n\}$. Each successive removal of $\eta_{i}^{*}$ together with $\eta_{i}$ from $\Delta$ for $i=1,2, \ldots, k$ is a collapse step. The cells left are $\Delta^{\prime \prime}=\{\eta \in \Delta \mid \eta(v)=\{n\}\}$. Finally, there is a bijection between the face posets of $\Delta^{\prime}$ and $\Delta^{\prime \prime}$ by extending each $\eta \in \Delta^{\prime}$ with $\eta(v)=\{n\}$.

The main use of Lemma 2.1 is when $I^{\prime}=\emptyset$. Then $n \notin \eta(w)$ for all $\eta \in \Delta^{\prime}$ and $w \in V(G) \backslash I$, so $\Delta^{\prime}=\operatorname{Hom}\left(G \backslash I, K_{n-1}\right)$. Another way to prove the lemma is to use discrete Morse theory [5].

Lemma 2.2 (Nerve Lemma, 2, Theorem 10.6(ii)], 3]). Let $\Delta$ be a regular cell complex, and $\left(\Delta_{j}\right)_{j \in J}$ a family of subcomplexes such that $\Delta=\bigcup_{j \in J} \Delta_{j}$ and every nonempty finite intersection $\Delta_{j_{1}} \cap \Delta_{j_{2}} \cap \cdots \cap \Delta_{j_{t}}$ is $(m-t+1)$-connected. Then $\Delta$ is $m$-connected if and only if the nerve $\mathcal{N}\left(\Delta_{j}\right)$ is m-connected.

Assume that $m \geq 0$. We will construct a family of subcomplexes such that $\Delta=\bigcup_{j \in J} \Delta_{j}$, all $\Delta_{j}$ are $m$-connected, and every intersection $\Delta_{j_{1}} \cap \Delta_{j_{2}} \cap \cdots \cap \Delta_{j_{t}}$ is $(m-1)$-connected for $t \geq 2$. If a complex is $(m-1)$-connected, then it is $(m-t+1)$-connected for $t \geq 2$. Since all intersections are nonempty, the nerve is a simplex, and $\Delta$ is $m$-connected.

Theorem 2.3. $\operatorname{Hom}\left(G, K_{n}\right)$ is $(n-\dot{\chi}(G)-1)$-connected.

Proof. We use induction on $\dot{\chi}(G)$ and on $n-\dot{\chi}(G)$. When $\dot{\chi}(G)=1, G$ has no edges, so $\operatorname{Hom}\left(G, K_{n}\right)$ is contractible, and in particular, $(n-\dot{\chi}(G)-1)$-connected. If $n-\dot{\chi}(G)=0$, then $n \geq \chi(G)$ so $\operatorname{Hom}\left(G, K_{n}\right)$ is nonempty, and $(n-\dot{\chi}(G)-1)-$ connected.

For all $I \in \mathcal{I}$, let $\Delta_{I}=\left\{\eta \in \operatorname{Hom}\left(G, K_{n}\right) \mid n \in \eta(i) \Rightarrow i \in I\right\}$, where $\mathcal{I}$ is the family of maximal independent subsets of $G$. Clearly, $\operatorname{Hom}\left(G, K_{n}\right)=\bigcup_{I \in \mathcal{I}} \Delta_{I}$. By 
Lemma 2.1 the complex $\Delta_{I}$ is homotopy equivalent to $\operatorname{Hom}\left(G \backslash I, K_{n-1}\right)$, which is $((n-1)-(\dot{\chi}(G)-1)-1)$-connected by Lemma 1.4 and induction. If $\mathcal{I} \supseteq \mathcal{I}^{\prime} \neq \emptyset$, then $\bigcap_{I \in \mathcal{I}^{\prime}} \Delta_{I}=\left\{\eta \in \operatorname{Hom}\left(G, K_{n}\right) \mid n \in \eta(i) \Rightarrow i \in \bigcap_{I \in \mathcal{I}^{\prime}} I\right\}$ is homotopy equivalent to $\operatorname{Hom}\left(G \backslash\left(\bigcap_{I \in \mathcal{I}^{\prime}} I\right), K_{n-1}\right)$ by Lemma 2.1, and $((n-1)-\dot{\chi}(G)-1)$-connected by Lemma 1.3 and induction. By the Nerve Lemma we are done.

Corollary 2.4. $\operatorname{Hom}\left(G, K_{n}\right)$ is $(n-d-2)$-connected.

Proof. Lemma 1.2 states that $\dot{\chi}(G) \leq d+1$.

\section{ACKNOWLEDGEMENTS}

We thank Sonja Čukić, Dmitry Kozlov, and the referee for their comments.

\section{REFERENCES}

1. E. Babson, D.N. Kozlov, Complexes of graph homomorphisms. Israel J. Math. 152 (2006), 285-312.

2. A. Björner, Topological Methods, in: "Handbook of Combinatorics" (eds. R. Graham, M. Grötschel, and L. Lovász), North-Holland, 1995, 1819-1872. MR1373690 (96m:52012)

3. A. Björner, L. Lovász, S.T. Vrećica, R.T. Živaljević, Chessboard complexes and matching complexes, J. London Math. Soc. (2) 49 (1994), 25-49. MR.1253009 (95c:52021)

4. S. Lj. Čukić, D.N. Kozlov, Higher connectivity of graph coloring complexes, Int. Math. Res. Notices. 2005:25 (2005), 1543-1562. MR2152894

5. R. Forman, Morse theory for cell complexes, Adv. Math. 134, no. 1, (1998), 90-145. MR 1612391 (99b:57050)

6. D.N. Kozlov, Chromatic numbers, morphism complexes, and Stiefel-Whitney characteristic classes, 67 pages, to appear in Geometric Combinatorics, IAS/Park City Mathematics Series 14, American Mathematical Society, Providence, RI; Institute for Advanced Study (IAS), Princeton, NJ.

Department of Computer Science, Eidgenössische Technische Hochschule, Zürich, SWITZERLAND

E-mail address: engstroa@inf.ethz.ch 